\title{
Detection of Non-palpable Prostate Cancer. A Mathematical and Laboratory Model
}

\author{
H. J. STRICKER, L. J. RUDDOCK, J. WAN and W. D. BELVILLE \\ Section of Urology, University of Michigan Medical Center, Ann Arbor, USA
}

\begin{abstract}
Summary-Prostate cancer is currently the most commonly diagnosed cancer among males in the United States. As technology improves and the search for this enigmatic condition intensifies, we are detecting greater numbers of non-palpable tumours. These tumours are generally treated aggressively, given the uncertainty of their behaviour, but this approach may be over-zealous for small volume disease. The likelihood of detecting any cancer volume can be derived from Bayes' theorem of conditional probability. A laboratory model using coloured clay was created to contrast tumour volumes of $2.5,5$ and $20 \%(n=75)$. Six random systematic biopsies were then taken from each model in a blind fashion; $36 \%$ of the $2.5 \%, 44 \%$ of the $5 \%$ and all of the $20 \%$ models had at least 1 positive biopsy. Twenty-two of the 25 models representing $20 \%$ tumour had 3 or more biopsy cores positive. These data suggest that low volume disease with low biological potential will be found by random biopsy as the mathematical probability predicts. The high incidence of occult prostate cancer in the older population makes this a worrying observation. Also, and perhaps more important, there is a direct correlation between the volume of disease and the number of positive biopsies. This correlation is easily seen in both models and may allow for an estimation of tumour volume. This ability to estimate tumour volume may be a useful clinical tool that helps to guide therapy and assess prognosis.
\end{abstract}

Prostatic cancer is now the most common clinically diagnosed cancer among males in the United States (Optenberg and Thompson, 1990). This increased rate of detection is due in part to widespread screening, which has uncovered more early stage and non-palpable cancer. In 1981, Cantrell et al. described $\mathrm{A} 1$ disease as $\leq 5 \%$ cancer by volume with a Gleason score of $\leq 4$ (Cantrell et al., 1981). Of those individuals whose prostate glands had $\leq$ $5 \%$ cancer only $2 \%$ showed progression. None of their well differentiated tumours advanced and only $11 \%(5 / 47)$ of those with Gleason scores 5 or 6 progressed. This study reaffirmed that stage A prostate cancer generally behaves in a benign manner provided it is not high grade and, together with other studies, supports the expectant treatment of individuals with low volume/grade carcinoma found on transurethral resection of the prostate

Accepted for publication 8 April 1992
(TURP). This management is justified since the 15 year survival with Al disease is similar to that of age-matched controls (Hanash et al., 1972). Those individuals with $\mathrm{A} 2$ (high volume or high grade) disease, however, are generally directed towards aggressive therapy.

Given increased public awareness and the intensified search, smaller volumes of cancer are being found which would previously have remained undetected. Nevertheless, patients with any cancer whatsoever found on needle biopsy are, seemingly by reflex, treated in an aggressive manner. The apparent basis for this practice is the belief that $\mathrm{Al}$ disease is unlikely to be detected by needle biopsy and the inability to distinguish it from A2 disease pre-operatively.

Given these unknowns, we have attempted to determine the incidence of a positive needle biopsy for a series of known cancer volume relationships. A mathematical model based on Bayesian statistics of conditional probability was used to derive 
diagnostic probabilities. A laboratory model was then created to contrast volumes of $2.5 \%$ and $5 \%$ cancer representing stage $\mathrm{A} 1$ disease, with a volume of $20 \%$ cancer representing stage $\mathrm{A} 2$ disease.

\section{Materials and Methods}

Mathematical probability was used to determine the likelihood of detecting cancer for any number of biopsies. This relationship is Bayes' theorem of conditional probability, which is a derivative of the binomial theorem (Woolson, 1981):

$$
\operatorname{Pr}(\mathrm{S}+/ \mathrm{C}+)=1-(1-\mathrm{p})^{\mathrm{n}}
$$

where $\operatorname{Pr}(\mathbf{S}+/ \mathbf{C}+)$ represents the probability that the sequence of biopsies is positive given that the patient has cancer, $p$ stands for the volume percent of the prostate that is malignant and $\mathrm{n}$ represents the number of biopsies in the sequence. The sequence is defined as positive if any one or more of the biopsies in the sequence is positive. This classic model deals with whole numbers and is valid if 2 assumptions are met: both the distribution of cancer and the biopsies must be random. While we are aware that no clinical event is ever truly random, the known multifocal nature of prostate carcinoma allows it to be considered as such for practical purposes. The method of "systematic random" biopsy is also not mathematically random but the inherent technical variability therein further supports this assumption.

The laboratory model was created from contrasting colours of clay: one designated as normal prostate tissue and the other as carcinoma. The normal tissue clay was sectioned into 39,38 and $32 \mathrm{ml}$ volumes and the clay designated as malignant was measured into 1,2 and $8 \mathrm{ml}$ volumes. These 3 groups of 25 each represented cancer volumes of $2.5,5.0$ and $20 \%$ respectively. The cancer clay was divided into 6 various sized foci and the prostate clay was broken into similar size pieces. Both were placed in airtight bags to ensure the volume would not change as a result of moisture loss. They were then placed in a freezer for 24 to $48 \mathrm{~h}$, which allowed for more even mixing. Their contents were placed in a bowl and stirred with an electric hand mixer for approximately $1 \mathrm{~min}$. The clay particles were then emptied into a small container and blindly packed roughly into the shape of a prostate. These "prostates" were then wrapped in aluminum foil and put back in the freezer for another 24 to $48 \mathrm{~h}$. Six random systematic biopsies were subsequently taken, from the apex to the base, as described by Hodge et al. (1989). A biopsy was read as positive if any amount of the cancer colour could be detected by visual inspection. A cross-sectioned clay model "prostate" is shown in Figure 1.

\section{Results}

The results from calculating Bayes' theorem with the number of biopsies (n) $=1-6$ and the percent cancer $(p)=1-5 \%, 10 \%, 20 \%, 25 \%$ and $50 \%$ are indicated in Table 1. The calculations show that as the number of biopsies or the percent volume cancer increases, so does the probability of finding positive histology. The mathematical model predicts that $26.5 \%$ of glands with $5 \%$ cancer will have at least 1 of 6 positive biopsies. Even if only 4 are taken, almost $20 \%$ will show cancer.

Of the 25 clay prostates with $2.5 \%$ cancer, 9 had a positive biopsy, all of these showing only 1 of the 6 biopsies to be positive. In other words, of the 150

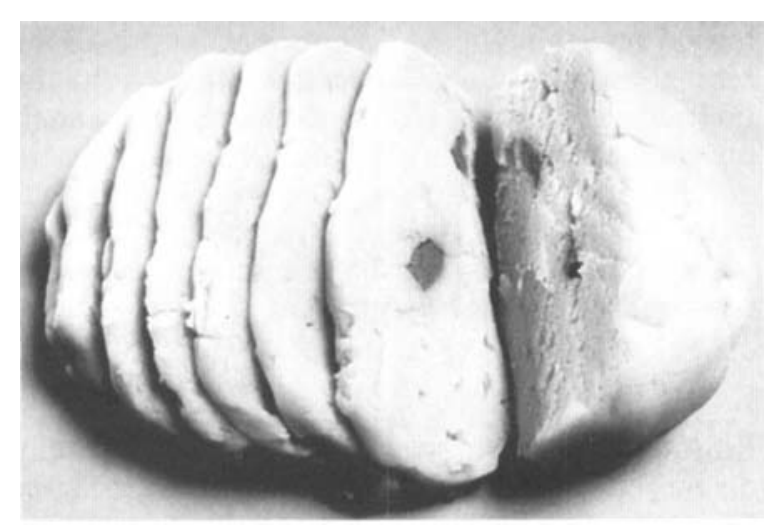

Fig. 1 A cross-sectioned model prostate.

Table 1 Probability of at Least One Positive Biopsy From Bayes' Theorem

\begin{tabular}{lllllll}
\hline \multirow{2}{*}{$\begin{array}{l}\text { \% Volume } \\
\text { cancer }\end{array}$} & \multicolumn{2}{l}{ Na. of biopsies } \\
\cline { 2 - 7 } & 1 & 2 & 3 & 4 & 5 & 6 \\
\hline $\mathbf{1}$ & 0.010 & 0.020 & 0.030 & 0.039 & 0.049 & 0.059 \\
$\mathbf{2}$ & 0.020 & 0.040 & 0.059 & 0.078 & 0.096 & 0.114 \\
$\mathbf{3}$ & 0.030 & 0.059 & 0.087 & 0.115 & 0.141 & 0.167 \\
$\mathbf{4}$ & 0.040 & 0.078 & 0.115 & 0.151 & 0.185 & 0.217 \\
$\mathbf{5}$ & 0.050 & 0.098 & 0.143 & 0.185 & 0.226 & 0.265 \\
$\mathbf{1 0}$ & 0.100 & 0.190 & 0.271 & $\mathbf{0 . 3 4 4}$ & 0.410 & 0.469 \\
$\mathbf{2 0}$ & 0.200 & 0.360 & 0.488 & 0.590 & 0.672 & 0.738 \\
$\mathbf{2 5}$ & 0.250 & 0.438 & 0.578 & 0.684 & 0.763 & 0.822 \\
$\mathbf{5 0}$ & 0.500 & 0.750 & 0.875 & 0.938 & 0.969 & 0.984 \\
\hline
\end{tabular}


Table 2 Laboratory Model Results

\begin{tabular}{llll}
\hline $\begin{array}{l}\text { No. of } \\
\text { positive } \\
\text { biopsies }\end{array}$ & \multicolumn{3}{c}{ Percent volume cancer } \\
\cline { 2 - 4 } & $2.5 \%$ & $5.0 \%$ & $20.0 \%$ \\
\hline $\mathbf{0}$ & 16 & 14 & - \\
$\mathbf{1}$ & 9 & 9 & - \\
$\mathbf{2}$ & - & 2 & 3 \\
$\mathbf{3}$ & - & - & 13 \\
$\mathbf{4}$ & - & - & 5 \\
$\mathbf{5}$ & - & - & 4 \\
$\mathbf{6}$ & - & - & - \\
\hline
\end{tabular}

cores taken, only $6 \%$ were positive. Eleven or $44 \%$ of the $5 \%$ cancer model had at least 1 positive biopsy. Here, only 2 "prostates" had more than 1 positive biopsy and none had as many as 3 . For this volume $(5 \%)$ the total number of positive biopsies was 13 or $8.7 \%$.

In the $20 \%$ volume cancer group used to illustrate A2 disease, all of the prostates had at least 2 biopsies positive; 22 of these had 3 or more positive. A total of 85 of the $150(56.7 \%$ ) taken demonstrated the cancer colour. These results are shown in Table 2.

\section{Discussion}

The stimulus for this work arose from our growing concern over the increasing effort directed towards the detection of non-palpable prostate cancer. Since much non-palpable disease is low volume and has a good prognosis, aggressive treatment may not be appropriate (George, 1988; Johansson et al., 1989; Adolfsson et al., 1990; Graversen et al., 1990; Adolfsson and Carstensen, 1991). With more screening modalities and an intensified search, the rate of detection of smaller, clinically insignificant tumours is most likely to be affected. While both prostate specific antigen (PSA) and prostate ultrasound have many drawbacks in screening for carcinoma of the prostate, they often do lead to prostate biopsy (Hudson et al., 1989; Terris et al., 1991). If a biopsy then demonstrates carcinoma, it is presumed that the histology is the cause for the abnormal test. However, it is possible that the 2 events are unrelated and that the positive biopsy simply reflects the prevalence of the condition in the population. Step sections of prostates without evidence of clinical disease on examination from autopsy and radical cystectomy specimens have found prostate carcinoma in 30 to $40 \%$ of older men (McNeal, 1969; Montie et al., 1989). Most of these specimens showed low volume disease. Both the mathematical and laboratory model described herein demonstrate that low volume disease will be found in sizeable numbers by random biopsy.

The results obtained from the clay models parallel those derived from Bayes' theorem (Fig. 2). The consistently higher rate of detection from the clay model probably reflects simply the vagaries of small numbers. Regardless of the model used, it appears that low volume disease can be detected by random biopsy. In fact, cancer was found in $36 \%$ of the model with $2.5 \%$ cancer. Given the high prevalence of occult disease in the older population, these numbers are alarming. There is an enormous number of men in whom our current practice pattern will allow us to detect clinically insignificant adenocarcinoma. More challenging is the task of determining what therapy, if any, is best suited for these individuals. This work suggests that multiple random biopses may be helpful in estimating tumour volume, thus guiding therapeutic decisions recalling the behaviour of small volume disease found on TURP.

Gleason score can differentiate A1 and A2 disease with ample needle biopsy. A poorly differentiated biopsy makes the lesion at least $\mathrm{A} 2$ regardless of volume. Conversely, low grade histology is associated with low volume disease (Catalona and Scott, 1986). Further information regarding volume of disease can be provided by analysing the number of positive biopsies; $88 \%$ of the A2 model $(20 \%)$ had at least 3 positive biopsies, while none of the 50 models representing Al disease showed more than 2 positive and only 2 of 50 had more than 1 positive.

Recently, in an attempt to determine the ability of systematic biopsy to detect cancer in men with

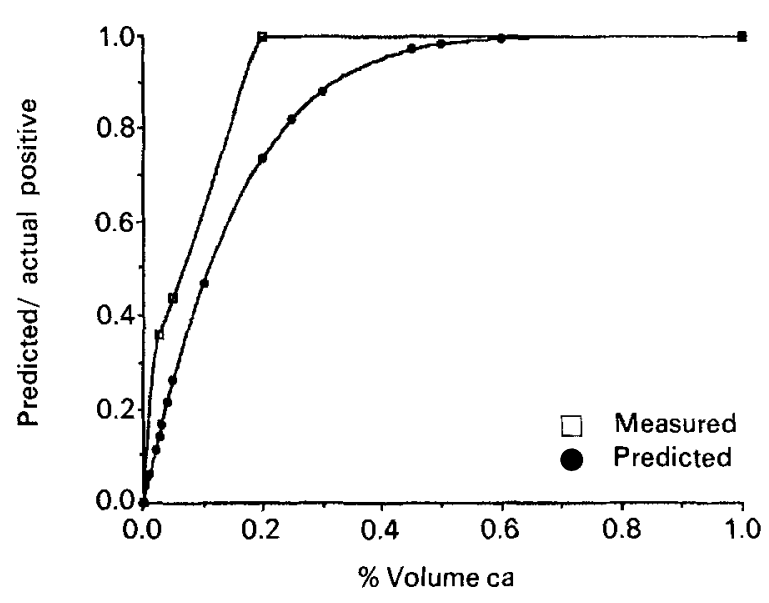

Fig. 2 Comparison of predicted calculated versus measured positive biopsies. 
BPH, Coplen et al. (1991) also found small volume disease using multiple biopsies. We believe the finding of well differentiated carcinoma in 1 of 6 cores may well represent stage A1 disease as shown in their report. It may be appropriate for a healthy 50 -year-old in this situation to be treated vigorously but an older individual may best be served by close follow-up, as are those with Al disease found by transurethral resection. Conversely, a patient with 5 or 6 positive biopsies almost certainly has large volume disease and requires therapy. We believe multiple random biopsies must be done judiciously, and in the proper setting will provide the clinician with useful data to buffer the uncertainties in dealing with this enigmatic disease.

\section{Acknowledgement}

The authors thank Ms Carrie Griffith for her initiative, patience and scholarly editorial input in the preparation of this manuscript.

\section{References}

Adolfsson, J. and Carstensen, J. (1991). Natural course of clinicially localized prostate adenocarcinoma in men less than 70 years old. $J$. Urol., 146, 96-98.

Adolfsson, J., Rönström, L., Carstensen, J. et al. (1990). The natural course of low grade, non-metastatic prostatic carcinoma. Br. J. Urol., 65, 611-614.

Cantrell, B. B., DeKlerk, D. P., Eggleston, J. C. et al. (1981). Pathological factors that influence prognosis on stage A prostatic cancer: the influence of extent versus grade. $J$. Urol, 125, 516-520.

Catalona, W. J. and Scott, W. W. (1986). Carcinoma of the prostate. In Campbell's Urology, ed. Walsh, P. C., Gittes, R. F., Perlmutter, A. D. and Stamey, T. A. Fifth edition. Volume 2. Chapter 32, pp. 1463-1534. Philadelphia: Saunders.

Coplen, D. E., Andriole, G. L., Yuan, J. J. J. et al. (1991). The ability of systematic transrectal ultrasound guided biopsy to detect prostate cancer in men with the clinical diagnosis of benign prostatic hyperplasia. J. Urol., 146, 75-77.

George, N. J. R. (1988). Natural history of localised prostatic cancer managed by conservative therapy alone. Lancet, 1. 494-497.

Graversen, P. H., Nielsen, K. T., Gasser, T. C. et al. (1990) Radical prostatectomy versus expectant primary treatment ir stages I and II prostatic cancer. Urology, 36 493-498.

Hanash, K. A., Utz, D. C., Cook, E. N. et al. (1972). Carcinoma of the prostate: a 15-year followup. J. Urol., 107, 450-453.

Hodge, K. K., MeNeal, J. E., Terris, M. K. et al. (1989). Random systematic versus directed ultrasound guided transrectal core biopsies of the prostate. J. Urol., 142, 71-75.

Hudson, M. A., Bahnson, R. R. and Catalona, W. J. (1989) Clinical use of prostate specific antigen in patients with prostate cancer. J. Urol., 142, 1011-1017.

Johansson, J.-E., Andersson, S.-O., Krusemo, U. B. et al. (1989). Natural history of localised prostatic cancer. A population. based study in 223 untreated patients. Lancet, 1, 799-803.

McNeal, J. E. (1969). Origin and development of carcinoma in the prostate. Cancer, 23, 24-34.

Montie, J. E., Wood, D. P., Pontes, J. E. et al. (1989). Adenocarcinoma of the prostate in cystoprostatectomy specimens removed for bladder cancer. Cancer, 63, 381-385.

Optenberg, S. A. and Thompson, I. M. (1990). Economics o: screening for carcinoma of the prostate. Urol. Clin. North Am. 17, 719-737.

Terris, M. K., Freiha, F. S., McNeal, J. E. et al. (1991). Efficacy of transrectal ultrasound for identification of clinicallv undetected prostate cancer. J. Urol., 146, 78-84.

Woolson, R. F. (1987). Basic probability concepts. In Statistica! Methods for the Analysis of Biamedical Data, ed. Barnett, C. Bradley, R. A. Hunter, J. S. et al. Chapter 3, pp. 59-64. New York: Wiley.

\section{The Authors}

H. J. Stricker, MD, House Officer IV.

L. J. Ruddock, Medical Student IV.

J. Wan, MD, Fellow.

W. D. Belville, MD, Associate Professor, Section Head.

Requests for reprints to: W. D. Belville, University of Michigar. Medical Center, Department of Surgery, Section of Urology. 1500 E. Medical Center Drive, TC-2918F, Ann Arbor, Michigan $48109-0330$, USA. 